\title{
La representación del acento en escritores principiantes muy jóvenes
}

\author{
Paulina Soni Limón y Sofía A. Vernon \\ Universidad Autónoma de Querétaro \\ (Texto recibido el 5 de diciembre de 2017; aceptado el 15 de junio de 2018) \\ DOI: http://doi.org/10.5565/rev/jtl3.770
}

Resumen: Esta investigación tuvo como objetivo indagar sobre los recursos gráficos de niños pre-alfabéticos y alfabéticos iniciales para marcar la diferencia entre pares de palabras que se diferenciaban por la posición de la tilde (por ejemplo, jugo/jugó). Se entrevistaron 77 niños de 4 a 7 años de la ciudad de Querétaro, México. Se dieron dos tareas: en la primera se les daba una palabra de cada par mínimo para que escribieran la otra. En la segunda, los niños elegían la palabra objetivo de entre dos palabras escritas, también pares mínimos. Los resultados obtenidos sugieren que: a) los niños mostraron diferentes maneras para diferenciar la posición de la tilde; a mayor nivel de escritura y nivel escolar, los recursos gráficos utilizados para diferenciar entre las palabras de los pares se van acercando a la convencionalidad; b) la comprensión de qué es la tilde aumenta conforme aumenta el nivel de escritura y la escolaridad.

Palabras clave: Adquisición de la escritura, alfabetización inicial, acentuación, tildación

\begin{abstract}
This study investigated the graphical resources used by pre-alphabetic and beginning alphabetic children to establish differences between pairs of words which were different only because of the placement of stress. 77 children from urban Mexican public schools (preschool, kindergarten, first and second grades) were interviewed. Two different tasks were assigned to the children: 1) writing using one of the words in the minimal pair as a model, and 2) choosing the written word that corresponded to the word uttered by the experimenter. Results suggest that a) The higher the school grade level and the higher the writing level, the more complex and conventional the graphic resources used by children become; b) as children advance towards a higher writing and school level, the more they seem to understand the role of stress marks, even if their understanding is by no means perfect.
\end{abstract}

Key words: Acquisition of written language, stress mark, early literacy, stress mark placement

Resumé : Cette recherche visait à étudier les ressources graphiques initiales des enfants pré alphabétiques et alphabétiques pour faire la différence entre les paires de mots qui différaient de la position de l'accent (par exemple, le jugo / jugó). Nous avons interviewé 77 enfants de 4 à 7 ans de la ville de Querétaro, au Mexique. Deux tâches ont été données: pour la première, on leur a donné un mot de chaque paire minimale pour qu'ils écrivent l'autre mot. Dans la seconde, les enfants ont choisi le mot cible entre deux mots écrits, ainsi que des paires minimales. Les résultats suggèrent que: a) les enfants ont montré différentes façons de différencier la position d'accent; selon le niveau d'écriture et le 
niveau scolaire plus élevé, les ressources graphiques utilisés pour différencier entre les mots des paires s'approchent à la conventionnalité; b) la compréhension de ce qu'est le tilde augmente à mesure que le niveau d'écriture et de scolarisation augmente.

Mots-clés : Acquisition de l'écriture, alphabétisation initiale, accentuation, placement de l'accent

\section{Introducción}

Este artículo tiene como propósito explorar si los niños pre-alfabéticos o alfabéticos iniciales pueden decidir representar el acento tónico de las palabras y cómo lo hacen.

El acento gráfico en el español. En todas las palabras poli-silábicas del español hay una sílaba acentuada. Es decir, una sílaba de mayor intensidad acústica o sílaba tónica. En el español, el acento prosódico siempre recae sobre una vocal.

En español, el acento es libre y puede ocurrir en cualquiera de las últimas sílabas de la palabra. Según la posición del acento, las palabras pueden clasificarse en agudas u oxítonas, si el acento está en la última sílaba, como en "patín", "profesor"; palabras graves o paroxítonas, si está en la penúltima, como "conejo" y finalmente palabras con el acento en la antepenúltima sílaba, llamadas esdrújulas o proparoxítonas, como "título" y "matrícula" (Hualde, 2014).

El acento tiene como función primordial distinguir formas fonológicamente idénticas que varían según su prosodia y que poseen significados distintos, como es el caso de pares o tríos de palabras como médico, medico, medicó; depositó, depósito, deposito o ánima, anima.

En la escritura, el acento se marca en algunas palabras con una pequeña raya oblicua (tilde) sobre la vocal prominente en la sílaba tónica. Además de permitir la distinción de palabras que de otra manera serían idénticas, como papa y papá, el acento gráfico u ortográfico tiene la función de indicar qué sílaba de una palabra debe recibir la mayor intensidad acústica y la de distinguir la función gramatical y el significado de palabras que se pronuncian de manera idéntica (en general para distinguir pronombres y adjetivos como "tú fuiste" vs "tu mamá").

No todas las palabras escritas se acentúan. Hay una especie de "economía de esfuerzo" (Gómez Fernández, 1979), así que la tilde se reserva para marcar aquellos casos que se desvían de los patrones generales de acentuación léxica (Hualde, 2014). Las reglas generales para la acentuación gráfica son:

a) Los monosílabos no llevan tilde, con excepción de la marca diacrítica para hacer distinciones como la que aparece en el punto anterior. 
b) Sólo se tildan las palabras agudas que terminan en vocal, o en " $n$ " o en "s".

c) Las palabras graves que terminan en letras que no sean vocal, "n" o "s" siempre se tildan.

d) Todas las palabras esdrújulas se tildan.

Estudios sobre acentuación gráfica. La acentuación gráfica es un aspecto importante de la ortografía del español y uno de los retos más difíciles para los estudiantes. En 2008, el INEE (Instituto Nacional para la Evaluación de la Educación) evaluó a 13 mil 807 alumnos mexicanos provenientes de diferentes tipos de escuelas (urbanas públicas y privadas, de educación indígena y rurales públicas) a partir de la escritura de los textos completos y con significado social producidos como parte de los Exámenes de la Calidad y el Logro Educativos (EXCALE) de español en la parte relativa a expresión escrita. El error ortográfico más común en los alumnos de tercero y sexto de primaria, así como en los alumnos de tercero de secundaria, fue la acentuación gráfica y en especial la omisión de la tilde en palabras agudas.

Urria (1988) también muestra que uno de los errores que más cometen los niños de quinto año de educación básica es la omisión de la tilde y la tildación de una vocal que no pertenece a la sílaba tónica.

La mayor parte de los trabajos anteriores analizan las desviaciones que los estudiantes de diferentes niveles educativos hacen de la norma (Báez, 2000; Sánchez Molina, 2003; Bolet, 1999). Son pocos los trabajos que intentan explorar las hipótesis que hacen los estudiantes sobre la tilde.

La investigación realizada por Vernon y Alvarado (2014), donde se trabajó con 552 niños y jóvenes entre tercer grado de primaria y tercer grado de secundaria de escuelas públicas y privadas se pedía a los participantes que subrayaran la palabra dicha por el investigador (una de tres palabras en contraste que se mostraban escritas del tipo MÉDICO/MEDICO/MEDICÓ) y, en otra tarea, que circularan "la parte de estas palabras que suenen más fuerte". Se observó que:

a) Los chicos de tercero y cuarto en ambos tipos de escuela y los de escuela pública hasta tercero de secundaria $\left(9^{\circ}\right)$ tienen dificultades para colocar la tilde en la vocal tónica, aun cuando haya un contraste de significado entre palabras.

b) La capacidad para localizar la sílaba tónica parece estar ligada a la escolaridad y, probablemente, a la conciencia metalingüística. 
c) La tilde parece no funcionar como marca prosódica para los niños de tercer y cuarto grado en las escuelas privadas ni para la mayoría de niños de escuelas públicas, independientemente del grado.

d) Para los niños más pequeños de escuelas públicas, localizar "la parte que suene más fuerte" no equivalió a encontrar la sílaba tónica. Muchos parecían buscar alguna propiedad segmental y subrayaban consonantes como R.

La presente investigación retoma y amplía los estudios de Vaca (1983 y 1997), para verificar si existe desarrollo temprano de este aspecto ortográfico, tan importante en el español. En estos trabajos, Vaca dio una tarea de tildación a niños de primero y segundo de primaria. El autor pidió a los participantes escribir dos palabras que sólo eran diferentes por la presencia o ausencia de tilde. Los resultados mostraron que los niños utilizan recursos no convencionales para representar las diferencias. Reporta el uso de mayúsculas, duplicación de letras y alternancia gráfica.

Esta investigación tuvo como propósito investigar si los niños de 4 a 7 años (2o de preescolar a 20 de primaria) eran capaces de:

a) representar la diferencia entre pares mínimos de palabras que se diferencian por la posición del acento y

b) usar la información provista por la tilde para diferenciar lo que decía en palabras contrastadas gráficamente.

Las hipótesis fueron que los niños podrían marcar las diferencias entre las palabras de maneras no convencionales y que las respuestas más convencionalizadas se incrementarían a la par de la escolarización. Asimismo, supusimos que la capacidad para comprender qué es la tilde y su uso aumentaría conforme aumentara el nivel de escritura de los niños y su escolaridad.

\section{Método}

Población. La muestra estuvo constituida por 77 participantes (38 niños y 39 niñas) entre los 4 y los 7 años. Los participantes asistían a escuelas públicas del estado de Querétaro, México. Del total, 16 estaban en el $2^{\circ}$ grado de preescolar (edad promedio en meses, $\mathrm{M}=57.4 ; \mathrm{SD}=3.5$ ), 31 en tercero de preescolar $(\mathrm{M}=67.3 ; \mathrm{SD}=7.5), 15$ en primer año de primaria $(\mathrm{M}=80.7 ; \mathrm{SD}=3.1)$ $y$ otros 15 en el segundo año de primaria $(M=93.4 ; S D=2.9)$. 
En todo momento se resguardó la confidencialidad de los participantes. Se pidió permiso a cada escuela (directores y docentes a cargo). Los padres de familia dieron su consentimiento para que los niños participaran en el estudio. Se cuidó también que los niños estuvieran de acuerdo en participar.

La muestra se eligió por un muestro no probabilístico por cuotas. Los criterios para la inclusión de participantes consistieron en que fueran niños hispanohablantes nativos, cursando los grados mencionados por primera vez. Se excluyó a niños con reporte de problemas de aprendizaje, lenguaje, audición o conducta.

Procedimiento: Escritura. Esta primera prueba sirvió para verificar el nivel de escritura de cada participante. A cada niño se le pidió que escribiera, en ausencia de modelos, 5 palabras con diferente número de sílabas: MARIPOSA, VENADO, PERICO, SAPO, MAR (adaptado del dispositivo experimental de Ferreriro y Teberosky, 1979). Los niños se clasificaron en 5 grupos según las escrituras que cada uno produjo: 1) escrituras presilábicas (PS); 2) silábicas sin predominio de valor sonoro convencional (SSVSC); 3) silábicas con predominio de valor sonoro convencional (SCVSC); 4) silábicas-alfabéticas (SA) y 5) alfabéticas iniciales (A).

Escritura de pares mínimos. Esta tarea tuvo por objetivo identificar si los niños podían representar la diferencia acentual entre las palabras y, las maneras que utilizaban para hacerlo (convencional o no). Se mostró a los niños la escritura de una de las dos palabras de un par mínimo y se les pidió a los participantes la escritura de la otra. Para ambas se dio una oración y una imagen que las contextualizaba. Se utilizaron seis pares de tarjetas de 11 x 17.5 con las imágenes y 6 tarjetas de $8 \times 3 \mathrm{~cm}$ con las palabras escritas en mayúsculas.

Los pares usados y el orden de presentación, fueron los siguientes (en itálicas se indica la palabra que los niños debieron escribir): jugo/jugó; saco/ sacó; peso/ pesó; papá/ papa; picó/ pico; bebé/ bebe.

Lectura de pares mínimos. El objetivo de esta tarea fue saber si los niños reconocían la tilde de manera escrita, el uso que tiene. Para esta tarea se utilizaron cinco pares de tarjetas de $8 \mathrm{x}$ $3 \mathrm{~cm}$ y 1 par de 8 x $1.5 \mathrm{~cm}$ con las palabras escritas en mayúsculas.

Se mostraron a los niños dos tarjetas, cada una con una palabra del par mínimo escrito. Se les dijo a los niños, en un primer momento, que observaran las palabras escritas que estaban sobre la mesa e indicaran las diferencias que hubiera entre ellas. En un segundo momento se les leían las tarjetas, sin indicar en qué letrero decía qué palabra y se les preguntaba a los 
participantes ¿dónde dice determinada palabra de cada par? Y ellos elegían una palabra del par. Para evitar sugerir una respuesta, el entrevistador procuraba no tocar ni señalar las tarjetas una vez colocadas en la mesa. Luego se pedía al participante que justificara su elección para conocer en qué se había fijado.

Los participantes podían observar y manipular las palabras siempre y cuando no alteraran el orden de presentación. Si el orden se alteraba, se colocaban en el orden original. El orden de presentación fue el siguiente (las palabras en itálicas indican las que los niños debían identificar como objetivo y las palabras con un asterisco al lado indican que fueron las primeras que se leían al decir qué dice cada palabra. En las tarjetas, la tipografía de ambas era idéntica salvo por la tilde): POPÓ/POPO*; EJÉRCITO*/EJERCITÓ; BEBÉ/BEBE*; PAPÁ*/PAPA; SACO/ SACÓ*. Los pares se presentaban en este orden, uno por uno, con la primera palabra arriba y la segunda justo abajo para facilitar su comparación.

\section{Resultados}

En total, 36 de los niños mostraron estar en un nivel alfabético inicial y 41 niños en los niveles prealfabéticos. Los participantes se clasificaron en los niveles de escritura como se indica en la tabla 1.

Tabla 1. Frecuencia de participantes por nivel de escritura y grado

\begin{tabular}{ccccccc}
\hline \multirow{2}{*}{ Nivel escritura } & \multicolumn{2}{c}{ Grado } & & \multirow{2}{*}{ Total } & Porcentaje \\
\hline PS & $2^{\circ}$ preescolar & $3^{\circ}$ preescolar & $1^{\circ}$ primaria & $2^{\circ}$ primaria & & $13 \%$ \\
SSVSC & 3 & 7 & 0 & 0 & 10 & $13 \%$ \\
SCVSC & 8 & 2 & 0 & 0 & 10 & $13 \%$ \\
SA & 3 & 7 & 0 & 0 & 10 & $14.29 \%$ \\
A & 2 & 8 & 1 & 0 & 11 & $46.71 \%$ \\
Total & 0 & 7 & 14 & 15 & 36 & $100 \%$ \\
\hline
\end{tabular}

Escritura de pares mínimos. Una hipótesis era que los niños pre-alfabéticos y alfabéticos utilizarían diferentes recursos gráficos para marcar la diferencia escrita entre dos pares mínimos de palabras que diferían solo por la posición del acento. Para comprobar esta hipótesis se realizó la tarea de escritura de pares mínimos, donde se mostraba a cada entrevistado una tarjeta con un dibujo que ejemplificaba el uso de la palabra modelo. Se mostraba la palabra modelo escrita convencionalmente en mayúsculas en una tarjeta que se colocaba bajo la imagen correspondiente. 
Luego se pedía al participante que escribiera la palabra objetivo debajo de la tarjeta con la imagen de contexto.

Las producciones de los participantes fueron registradas y sus respuestas fueron clasificadas en cinco categorías de análisis ordenadas de menor a mayor complejidad:

Cambia toda la escritura. En esta categoría se incluyeron todas las escrituras que cambiaban totalmente las letras de la palabra a escribir. Del total de 462 respuestas, 59 fueron de este tipo.

Un ejemplo claro de esta categoría es lo mencionado por E ( $2^{\circ}$ de preescolar, edad 61 meses). En todos los ejemplos, la escritura del niño se indica entre corchetes:

Investigadora (I): Te voy a enseñar unas imágenes y te voy a enseñar una palabra y a ti te toca escribir otra. Fíjate la primera. Aquí está el jugo y aquí dice jugo (mostrando letrero e imagen). Acá tengo el niño que jugó (imagen). A ti te toca escribir jugó.

\section{Us Mons}

Imagen 1. Escritura de $E\left(2^{\circ}\right.$ de preescolar, edad 61 meses $)$

E: (Escribe [USMONS])

I: ¿Quedaron iguales o diferentes?

E: Diferentes.

I: ¿En qué son diferentes?

E: En todas las letras.

I: ¿Por qué va con letras diferentes?

E: Pues es que es otra palabra. Si la escribo con estas letras (señala letrero) va a decir lo mismo y entonces serían lo mismo. Así ya es otra cosa, es de que el niño juega con la pelota.

I: ¿En esta qué dice? (letrero).

E: Jugo.

I: ¿Y en la tuya?

E: Jugó.

Como se puede apreciar, E parece tratar de crear diferencias entre las dos escrituras para poder representar diferentes significados.

Mantiene la escritura original. En esta categoría se incluyeron las escrituras que mantenían las letras de la palabra y el orden de las letras que la investigadora presentaba para escribir la palabra objetivo. Del total de 462 respuestas, 94 fueron clasificadas en esta categoría. Pudimos distinguir dos subcategorías de respuesta: 
a) Escritura idéntica, en las que los participantes utilizaron las mismas letras del modelo presentado, sin alterar ni su forma ni su orden. De las 94 respuestas de esta categoría, 86 pertenecen a esta subcategoría.

b) Escrituras con cambio de orientación: Estas respuestas, poco frecuentes, muestran escrituras que mantenían las mismas letras, pero cambiaban la orientación de alguna de las letras, haciendo, por lo general, una rotación de alguna de las letras.

El siguiente fragmento de entrevista a AS ( $2^{\circ}$ preescolar, 59 meses) ejemplifica las respuestas más numerosas de esta categoría (escrituras idénticas):

I: Te voy a enseñar unas imágenes que ya viste y te voy a enseñar una palabra y a ti te toca escribir otra ¿sale? Fíjate la primera. Aquí está el jugo y aquí dice jugo (mostrando letrero e imagen). Acá tengo el niño que jugó, a ti te toca escribir jugó.

AS: Necesitamos las mismas letras (va copiando las letras del letrero) [JUGO]

I: ¿Y quedaron iguales o diferentes?

AS: Iguales.

I: Entonces, ¿Cómo le podemos hacer para que la tuya diga jugó?

AS: Pues hay que borrar las letras (borra las letras).

I: Ahora, entonces ¿qué letras necesitas para jugó?

AS: (ve el letrero y dice) Éstas (señalando las de JUGO). (Toma el letrero, lo mueve al otro dibujo). Es que si lo paso acá ya dice su nombre.

I: Oye, pero ¿son cosas iguales o diferentes?

AS: Diferentes, porque una es jugo y otra jugó futbol.

I: Y entonces ¿podemos escribir cosas diferentes con letras iguales?

\section{JUG०}

Imagen 2. Escritura de AS ( $2^{\circ}$ preescolar, 59 meses)

AS: Si (escribe [JUGO]).

I: Bueno, entonces ¿Ésta qué dice? (letrero)

AS: Jugo de naranja

I: ¿Y en la tuya?

AS: Jugó, del niño que jugó futbol.

Un ejemplo de la subcategoría b) es la siguiente, en la que la participante escribe de manera idéntica al modelo, pero rotando una de las letras:

\section{$B E B \Uparrow$}

Imagen 3. Escritura de $\mathrm{D}\left(2^{\circ}\right.$ de preescolar, 52 meses $)$ 
Como se puede ver en el ejemplo anterior, los participantes tuvieron en cuenta las semejanzas sonoras para escribir y usaron las mismas letras que el modelo, a la vez que tenían claridad sobre el cambio y diferencia (de intensidad y de significado) entre las palabras.

Uso de recursos no convencionales. Estas respuestas presentaban algún recurso gráfico no convencional para marcar, a la vez, las semejanzas sonoras y las diferencias tanto sonoras como de significado entre las dos palabras. De las 462 respuestas en total, 133 fueron clasificadas en esta categoría. Es posible distinguir dos subcategorías:

a) Quita letras a la palabra mostrada y/o cambia orden de letras (54 de las 133 respuestas).

b) Agrega, duplica y/o cambia alguna letra (79 de las 133 respuestas). Se agruparon estas respuestas porque los participantes solo modificaban una letra de la escritura.

El siguiente fragmento de entrevista con $\mathrm{H}\left(3^{\circ}\right.$ preescolar, 72 meses) ejemplifica la primera subcategoría:

H: (Escribe primero [SACO] para sacó. Luego ve que el letrero y su escritura son idénticas). Puedo borrar (borra toda la palabra y se vuelve a autodictar y escribe [SAOC]).

\section{$S A O C$}

Imagen 4. Escritura $\mathrm{H}\left(3^{\circ}\right.$ preescolar, 72 meses $)$

I: Sale, entonces. ¿Son iguales o diferentes?

H: Está igual en estas dos partes (señalando SA) pero en éstas está diferente, porque aquí está al revés (señalando OC).

I: ¿Por qué irá al revés?

$\mathrm{H}$ : Pues es que si la pongo como estaba es lo mismo y no se puede escribir con las mismas letras cosas diferentes y entonces va así.

I: Entonces ¿en ésta qué dice? (letrero).

H: Saco.

I: ¿Y en la tuya?

H: Sacó.

Como es posible observar, $\mathrm{H}$ estaba consciente de que dos palabras con las mismas letras dicen igual. Pudo notar, sin embargo, que hay semejanzas y diferencias sonoras y un cambio en el significado. La solución que dio $\mathrm{H}$ es usar las mismas letras con un pequeño cambio en el orden de las mismas.

Un ejemplo para la segunda categoría en la que se agrega, duplica y/o cambia alguna letra es el de V ( $3^{\circ}$ preescolar, 72 meses), que escribe JUGON (para jugó), SACOS (para sacó), PESOT (para pesó), PICON (para pico). 


\section{JUGON \\ Sacos \\ PESOT \\ PAPA \\ PICON}

Imagen 5. Escritura de $\mathrm{V}\left(3^{\circ}\right.$ preescolar, 72 meses $)$

Otro ejemplo es el de O ( $1^{\circ}$ primaria, 82 meses $)$, que escribe PESOSO para pesó, ya que "cuando dice pesó, suena la última más fuerte y por eso va otra". O escribe también JUGOro para jugó y sacoro para sacó.

\section{JUGoto Sacoto PESOSO}

Imagen 6. Escritura de $\mathrm{O}\left(1^{\mathrm{o}}\right.$ primaria, 82 meses $)$

De manera similar, JC ( $1^{\circ}$ primaria, 85 meses) y LS ( $2^{\circ}$ primaria, 96 meses $)$ escriben ambos JUGOO, SACOO y PESOO para jugó, sacó y pesó. En palabras de uno de ellos, "la o suena fuerte".

\section{Sacoo}

\section{pesso}

Imagen 7. $\mathrm{JC}\left(1^{\circ}\right.$ primaria, 85 meses $)$

Recursos ortográficos: En esta categoría se incluyeron todas las escrituras que presentaron el uso de lo que generalmente se consideran recursos ortográficos, como el uso de letras que representan mismos fonemas: $\mathrm{c}, \mathrm{k}$ y q; b y v, signos de puntuación o cambio de posición de la tilde. Del total de respuestas, 47 se clasificaron en esta categoría. Se establecieron tres subcategorías:

a) Cambiar por posible: Se incluyeron todas aquellas escrituras en las cuales los participantes cambiaban alguna de las letras de la palabra presentada por una que tuviera la misma correspondencia sonora, por ejemplo, s por c y z, j por g, etc. para poder escribir 
la nueva palabra. Por ejemplo, KG ( $2^{\circ}$ primaria, 91 meses) escribe [gugo] para jugó y explica "(son) diferentes porque ésta (señala letrero con escritura convencional de JUGO) lleva jota y la otra (la suya) lleva ge (...). Es que suenan como muy iguales, pero si las escribo con las mismas letras dicen lo mismo y con esta (señala g) se parece, pero ya es otra palabra". KG escribe zaco para sacó porque "como la z se parece a la s, pues así ya es otra palabra, pero suena igual". Hubo 34 respuestas de este tipo (de un total de 47 en la categoría 4).

\section{gogo}

\section{Zero}

Imagen 8. Escritura de $\mathrm{KG}\left(2^{\circ}\right.$ primaria, 91 meses $)$

b) Puntos, comas: Se incluyeron las escrituras que presentaron puntos o comas para marcar la diferencia entre una palabra y otra. Por ejemplo, A ( $2^{\circ}$ de primaria, 89 meses) escribe [saco] para sacó y papa, explicando, para esta última "Pues porque suena fuerte en la primera y para eso se pone ahí". Solamente hubo 2 respuestas de este tipo (de las 47 de la categoría 4).

\section{Sqco,}

papa

Imagen 9. Escritura de A ( $2^{\circ}$ de primaria, 89 meses $)$

c) Cambio de posición de la tilde: En esta subcategoría están incluidas todas las escrituras que colocaron tilde, pero en una letra que no es la correcta, siempre acentuando vocales, no consonantes ( 11 respuestas de esta subcategoría en total). Por ejemplo, V ( $1^{\circ}$ primaria, 77 meses) escribió [júgo] para jugó, [péso] para pesó, [sáco] para sacó, [pápa] para papa, [píco] para pico y [bébe] para bebe. Hubo 11 respuestas de este tipo, todas de niños con nivel A de escritura. 


\section{júgo \\ péso \\ sáco \\ pápa \\ píco}

Imagen 10. Escritura de $\mathrm{V}\left(1^{\circ}\right.$ primaria, 77 meses $)$

Correctas: Se consideraron todas aquellas escrituras que quitaran o pusieran correctamente la tilde en las palabras.

La Tabla 2 muestra la relación entre las respuestas obtenidas y el nivel de escritura de los participantes.

Tabla 2. Frecuencia de respuestas por categoría y nivel de escritura en la Tarea 1

\begin{tabular}{|c|c|c|c|c|c|c|c|}
\hline \multirow{2}{*}{ Categoría } & \multirow{2}{*}{$\begin{array}{c}\text { Sub } \\
\text { categoria }\end{array}$} & \multicolumn{6}{|c|}{ Nivel de escritura } \\
\hline & & $P S$ & SSVSC & $S C V S C$ & $S A$ & $A$ & TOTAL \\
\hline 1. Cambia todo & ------ & 35 & 18 & 6 & 0 & 0 & 59 \\
\hline \multirow[b]{2}{*}{ 2. Se mantiene } & $\begin{array}{l}2.1 \text { Se mantiene } \\
\text { sin cambio }\end{array}$ & 10 & 26 & 29 & 9 & 12 & 86 \\
\hline & $\begin{array}{l}2.2 \text { Se mantiene } \\
\text { con cambio de } \\
\text { orientación en } \\
\text { alguna letra }\end{array}$ & 4 & 3 & 0 & 1 & 0 & 8 \\
\hline \multirow{2}{*}{$\begin{array}{l}\text { 3. Recursos no } \\
\text { convencionales }\end{array}$} & $\begin{array}{c}3.1 \text { Quita, } \\
\text { cambia orden }\end{array}$ & 5 & 3 & 15 & 24 & 7 & 54 \\
\hline & $\begin{array}{c}3.2 \text { Agrega, } \\
\text { duplica, cambia } \\
\text { letra }\end{array}$ & 2 & 9 & 7 & 28 & 33 & 79 \\
\hline \multirow{3}{*}{$\begin{array}{l}\text { 4. Recursos } \\
\text { ortográficos }\end{array}$} & $\begin{array}{c}\text { 4.1 Cambia por } \\
\text { posible }\end{array}$ & 0 & 0 & 0 & 0 & 34 & 34 \\
\hline & $\begin{array}{l}4.2 \text { Comas } \\
\text { puntos }\end{array}$ & 0 & 0 & 0 & 0 & 2 & 2 \\
\hline & $\begin{array}{c}4.3 \text { Cambia la } \\
\text { posición del } \\
\text { acento }\end{array}$ & 0 & 0 & 0 & 0 & 11 & 11 \\
\hline \multirow{2}{*}{$\begin{array}{r}\text { 5. Correctas } \\
T\end{array}$} & ---- & 4 & 1 & 3 & 4 & 117 & 129 \\
\hline & & 60 & 60 & 66 & 66 & 216 & 462 \\
\hline
\end{tabular}

La tabla 2 muestra que conforme aumenta el nivel de escritura los recursos gráficos usados por los participantes fueron cada vez más complejos. Para saber con certeza sobre la evolución de las hipótesis que hicieron los participantes sobre cómo representar gráficamente el 
acento, se realizó una Chi cuadrada que arrojó un valor de $\left.\chi^{2}(16, N=462)=446.57, p=.0001\right)$, lo que indica que a mayor nivel de escritura los recursos que utilizan los participantes tienden a ser más complejos y cercanos a la convencionalidad.

En la Tabla 2 se puede ver que los participantes con nivel PS de escritura sobre todo y, en menor frecuencia los SSVSC, tienden a cambiar toda la escritura de la palabra objetivo. Por su parte, la respuesta más frecuente entre los participantes con niveles SSVSC y SCVSC fue mantener la escritura del modelo para la palabra objetivo. Los participantes con nivel SCVSC, y sobre todo los SA e incluso A fueron los que usaron más los recursos gráficos no convencionales como agregar o duplicar letras. Los participantes con nivel A fueron los únicos que utilizaron recursos ortográficos y dieron respuestas convencionales.

La tabla 3 muestra la relación entre el tipo de estímulo escrito (palabras graves, como saco, o agudas, como sacó) y las respuestas categorizadas.

Tabla 3. Frecuencia por categoría de respuesta y tipo de palabra.

\begin{tabular}{cccc}
\hline Recurso gráfico utilizado & $\begin{array}{c}\text { Niños escriben } \\
\text { jugó, sacó, pesó }\end{array}$ & $\begin{array}{c}\text { Niños escriben } \\
\text { papa, pico, bebe }\end{array}$ & Total \\
\hline Cambia todo & 29 & 30 & 59 \\
Se mantiene & 56 & 38 & 94 \\
Recursos no convencionales & 85 & 48 & 133 \\
Recursos ortográficos & 35 & 12 & 47 \\
Correctas & 26 & 103 & 129 \\
Total & 231 & 231 & 462 \\
\hline
\end{tabular}

$\mathrm{Al}$ analizar la Tabla 3 se puede observar que las tres primeras palabras que se presentaban como modelos escritos (sin tilde) suscitaron más respuestas tipo 3 (recursos no convencionales), mientras que las últimas tres palabras que se presentaban como modelo (con tilde) presentaron más respuestas correctas. Para saber si el tipo de palabra que se les pidió escribir a los participantes influyó en sus decisiones gráficas se corrió una Chi cuadrada y se obtuvo como resultado una $\chi^{2}(4, N=462)=70.97, p=.0001$, lo que indica que el tipo de recurso gráfico usado se vio influido por el tipo de palabra que se le mostró como estímulo y que se le solicitó escribir. Es importante mencionar que el orden de presentación de las palabras fue fijo.

Lectura de pares mínimos. Esta tarea buscaba conocer si la capacidad comprender qué es la tilde y su uso aumentaba conforme aumentaba la escolaridad y el nivel de escritura de los niños. 
Los primeros pares mínimos que fueron presentados tuvieron menos aciertos que los últimos tres pares (de las 229 respuestas correctas, 37 para popó, 39 para ejercitó, 51 para bebé, 50 para papá y 52 para saco). En lo general, se puede observar que más de la mitad de los participantes (del total de 77) fueron capaces de seleccionar correctamente la palabra objetivo.

La tabla 4 muestra las respuestas en relación con el nivel de escritura. Entre paréntesis se indica el número de participantes por nivel de escritura.

Tabla 4. Frecuencia y porcentaje de aciertos por nivel de escritura en la Tarea 2

Nivel de escritura $\quad \begin{aligned} & \text { Total de } \\ & \text { aciertos }\end{aligned} \begin{gathered}\text { Porcentaje parcial de } \\ \text { aciertos }\end{gathered} \quad$ Porcentaje acumulado

\begin{tabular}{ccccc}
\hline & Presilábico (10) & 27 & $11.79 \%$ & \\
SSVSC & 21 & $9.17 \%$ & $40.61 \%$ \\
Prealfabético & $(10)$ & & $10.04 \%$ & \\
& SCVSC & 23 & $9.60 \%$ & $59.39 \%$ \\
& $(10)$ & 22 & $59.39 \%$ & $100 \%$ \\
\hline \multirow{2}{*}{ Alfabético } & Silábico-Alfabético (11) & 136 & $100 \%$ & \\
& Alfabético (36) & 229 & & \\
& Total (77) & &
\end{tabular}

Como se observa en la Tabla 4, los participantes de los niveles PS, SSVSC, SCVSC y SA (prealfabéticos) tienen menos aciertos que los participantes alfabéticos. Los participantes alfabéticos son capaces de distinguir más acertadamente que la presencia o ausencia de la tilde cambia el significado de las palabras.

Después de realizar el análisis por ítem, se realizó el análisis por participante para explorar si la capacidad de comprender qué es la tilde y su uso aumenta conforme aumenta la escolaridad y el nivel de escritura.

Una vez obtenidos los estadísticos descriptivos, se realizó el análisis estadístico. Para realizarlo, se tomó el nivel de escritura (prealfabético y alfabético) y las respuestas correctas totales de cada participante. Como resultado se obtuvo una $t(74.624)=-5.692, p=.000$. El resultado indica que los participantes alfabéticos tienen en promedio significativamente más alto de respuestas correctas que los participantes prealfabéticos.

Para conocer si existía correlación o no entre el nivel de escritura y las respuestas de los participantes se corrió una correlación de Spearman. Se obtuvo un coeficiente de Spearman de $r$ 
(75) $=.466$ y $p=.01$ bilateral. Este resultado indica que la correlación es significativa. Por lo tanto, conforme el nivel de escritura de los participantes aumenta, se incrementa su capacidad para distinguir la tilde y para seleccionar correctamente la palabra objetivo.

Para corroborar lo anterior y ser más específicos en cuanto al nivel de escritura, se corrió una ANOVA de un factor con una prueba posthoc de Bonferroni, con un alfa de .05. Para esta prueba se tomó el nivel de escritura visto desde los 5 niveles (PS, SSVSC, SCVSC, SA y A) y el total de respuestas correctas. En ésta se obtuvo una $F(4,72)=8.292$, $p=.000$. Al analizar la prueba de Bonferroni se puede observar que los niveles PS, SSVSC, SCVSC y SA no tienen diferencias significativas entre ellos, pero si tienen diferencia altamente significativa con el nivel A. Es decir, corrobora que los participantes alfabéticos se desempeñan mejor en la tarea.

Una vez realizado el análisis estadístico para el nivel de escritura, se realizó una $t$ de Student de muestras independientes, obteniendo una $t(74.684)=-7.841, p=.000$. Los resultados indican que los participantes de primaria tienen en promedio más respuestas correctas que los participantes de preescolar.

También se realizó una correlación de Spearman, para la que se tomaron las respuestas totales de los participantes y su nivel de escolaridad (preescolar y primaria). Esta prueba arrojó una $r(75)=.650$ y $p=.01$. El resultado indica que la correlación es significativa, por lo que conforme aumenta la escolaridad aumenta la capacidad para distinguir la tilde y por lo tanto de seleccionar de manera correcta la palabra objetivo.

Para obtener resultados con mayor certeza, se decidió correr una ANOVA de una vía con una prueba posthoc de Bonferroni con un alfa de .05 para conocer con mayor precisión si el grado influía en las respuestas de los alumnos. Se tomó el grado escolar $\left(2^{\circ}\right.$ y $3^{\circ}$ de preescolar, $1^{\circ}$ y $2^{\circ}$ de primaria) y el total de respuestas totales de los participantes. Como resultado se obtuvo una $F(3,73)=18.726, p=.000$. Este resultado indica que la diferencia entre grupos es altamente significativa. Se pudo apreciar que todos los grupos presentan alta diferencia significativa entre ellos, por lo cual también se corrobora que conforme aumenta la escolaridad y el grado escolar, los participantes tienden a tener un mejor desempeño y elección correcta de la palabra objetivo.

\section{Discusión}

Los resultados encontrados en la tarea de escritura de pares mínimos mostraron que a mayor nivel de escritura y escolaridad los recursos gráficos utilizados por los participantes para marcar el 
acento fueron cada vez más cercanos a la convencionalidad ortográfica. Más de la mitad de los participantes con niveles de escritura PS y casi una tercera parte de los participantes SSVSC (los menos avanzados en el nivel de escritura) cambiaron totalmente la escritura de la palabra modelo. Los niños de este nivel probablemente tengan muy presente la hipótesis de diferenciación entre escrituras (Ferreiro, 1997) que indica que para que dos escrituras "digan" distinto, deben estar escritas de manera distinta. Estos niños justifican sus escrituras argumentando que como son cosas diferentes no pueden llevar las mismas letras. Esto coincide con los hallazgos de Vaca (1997).

A diferencia de los participantes mencionados con anterioridad, poco menos de la mitad de los participantes con niveles de escritura SSVSC y SCVSC mantuvieron las mismas grafías de la palabra que se les presentó para escribir la palabra objetivo. Probablemente reconocen las diferencias de significados entre cada una de las palabras, pero son incapaces de encontrar algún recurso gráfico que les permita marcar esta diferencia en la escritura a la vez que marcar las semejanzas sonoras. Es decir, los niños que empezaban a tener correspondencia sonora en la escritura parecieron privilegiar la representación de las semejanzas sonoras sobre las diferencias de significado. Algunos de los participantes con respuestas tipo 2 hicieron intentos de conciliar este juego de semejanzas y diferencias conservando las mismas letras, pero orientando alguna de ellas de manera diferenciada al modelo.

La mayoría de los participantes con nivel de escritura SA y algunos A, que probablemente habían empezado a darse cuenta de que hay distintas altenativas para representar un mismo fonema y de que existen otros recursos gráficos que no son letras, utilizaron con más frecuencia recursos que hemos llamado "no convencionales", como agregar o duplicar letras para representar el acento. Sus respuestas muestran que estos niños saben que hay que mantener la sonoridad y las letras que representan los valores sonoros, pero son sensibles a las diferencias acentuales. Las marcan aumentando una consonante o duplicando la vocal para mostrar una mayor intensidad. Los recursos mencionados en el párrafo anterior recuerdan lo que Quinteros (2004) denominó "letra comodín o comodín silábico" (pag. 41), aunque la autora utilizó este término para hacer referencia al uso de letras no pertinentes por su valor sonoro convencional para "marcar el lugar" de la letra pertinente pero desconocida para los niños en ese momento. En este caso, los niños ponían una letra no pertinente a la palabra para marcar lo que les faltó por representar (el acento). En sus justificaciones mencionaban que al final sonaba más o sonaba 
diferente y la marcación que hacían de lo diferente parecía privilegiar la cantidad (o intensidad) más que la calidad. Este tipo de respuestas también recuerda al trabajo de Alvarado y Ferreiro (2002), quienes encontraron que hay niños que utilizan "números comodines" para marcar aquellos números que aún no saben representar.

Por último, los participantes A, principalmente los de primaria, fueron los únicos que utilizaron recursos como cambiar una letra por otra posible, colocar el acento en otra posición o usar puntos y comas. El uso de este tipo de recursos no convencionales puede relacionarse con lo que Quinteros (2004) denominó "letra sustituta”. La autora mostró el uso de una letra para marcar un sonido consonántico en la cadena oral pero para el que aún no conocen la letra pertinente. La "letra sustituta" usualmente es una letra que representa muchos de los mismos rasgos fonológicos de la letra objetivo (c por k, j por g). En este trabajo los niños alfabéticos usan lo que llamamos "recurso ortográfico de alternancia gráfica" cuando son capaces de identificar las diferencias acentuales en la oralidad, pero no cuentan con el recurso gráfico convencional para poder marcar las diferencias en la escritura.

Los participantes de primaria que utilizaron comas o puntos para marcar la diferencia acentual, al igual que los que tildaron la letra o sílaba incorrecta, están un paso más avanzados que los que utilizan la alternancia gráfica, pues ya consideran que la diferencia acentual no se representa con letras sino que se trata de algo ajeno a lo alfabético y que esta diferencia se representa por marcas diferentes.

En la tarea en la que se les pedía identificar el letrero con la palabra objetivo los resultados muestran que tanto los participantes alfabéticos, sobre todo los de primaria, también tienen mayores respuestas correctas que los participantes prealfabéticos y de preescolar.

Siempre se ha supuesto que el aprendizaje de la ortografía acentual se da gracias a una serie de repeticiones de palabras o a la memorización de las reglas de acentuación. Sin embargo, como se ha visto a lo largo de esta investigación, el aprendizaje de la atildación es un proceso de construcción complejo que implica reflexiones metalingüísticas alrededor del acento tónico, su posición, las marcas necesarias para representarlo, etc. Esta reflexión probablemente surja por la necesidad misma de escribir en contextos comunicativos demandantes.

Aunque la presente investigación da luz sobre algunas de la hipótesis que los niños hacen con respecto a la tilde, sería conveniente realizar investigaciones que permitan ahondar y clarificar las ideas que los niños ponen en juego al intentar usar o reconocer esta marca gráfica. 
Por último, es importante mencionar que la generalización de resultados de esta investigación está sujeta a ciertas limitaciones. Por ejemplo, sería necesario, en posteriores investigaciones, revisar la frecuencia de uso de las palabras presentadas.

\section{Referencias}

Alvarado, M., y Ferreiro, E. (2002). Four and five-year-old children writing with two-digit numbers. Revista di Psicolingüistica Applicata, 2(3), 23-27

Báez, G. E. (2000). Errores de acentuación gráfica más frecuentes en escolares de $6^{\circ}$ de primaria del D.F. Lingüistica Mexicana, 1, 127-142. Recuperado de http://amla.org.mx/linguistica_mexicana/Vol_I_1/2000010111a.pdf

Bolet, F. J. (1999). Errores de tildación en la redacción de textos narrativos y expositivos. Revista Paradigma. Recuperado de http://revistas.upel.edu.ve/index.php/paradigma/article/view/2948/1376

Ferreiro, E. (1997). Alfabetización, Teoría y práctica. México: Siglo XXI Editores.

Ferreiro, E., y Teberosky, A. (1979). Los sistemas de escritura en el desarrollo del niño. México: Siglo XXI.

Gómez Fernández, D. (1979). Las normas actuales de acentuación ortográfica: desarrollo y puntualizaciones. Cauce: Revista de Filología y su Didáctica, 2, 33-114. Recuperado de http://cvc.cervantes.es/literatura/cauce/pdf/cauce02/cauce_02_004.pdf

Hualde, J. I. (2014). Los sonidos del español. Cambridge: Cambridge University Press. DOI: https://doi.org/10.1017/CBO9780511719943

Quinteros, G (2004). La libertad de las letras. En A. Pellicer y S. Vernon (coord.), Aprender y enseñar la lengua escrita en el aula (pp. 41-70). México: Aula nueva SM.

Sánchez Molina, E. (2003). El problema de la acentuación y solución para el correcto desempeño académico y profesional (tesis de maestría). Universidad de las Américas, México. Recuperado de http://catarina.udlap.mx/u_dl_a/tales/documentos/mll/sanchez_m_e/portada.html

Urria, A. (1988). Una experiencia pedagógica sobre ortografía acentual. Lectura y Vida, 9(2), 10 16. Recuperado de http://www.lecturayvida.fahce.unlp.edu.ar/numeros/a9n2/09_02_Urria.pdf

Vaca, J. (1983). Ortografia y significado. Lectura y Vida, 4(1). Recuperado de http://www.lecturayvida.fahce.unlp.edu.ar/numeros/a4n1/04_01_Vaca.pdf

Vaca, J. (1997). Lo no alfabético del sistema de escritura: ¿Qué piensa el escolar? México: DIECINVESTAV.

Vernon, S., y Alvarado, M. (2014). El desarrollo de la acentuación gráfica en niños y jóvenes mexicanos. Revista Mexicana de Investigación Educativa, 18(56), 141-157. 
Información de las autoras:

Paulina Soni Limón es Licenciada en Psicología Educativa, con especialidad en Aprendizajes Escolares (Universidad Pedagógica Nacional, México). Laboró como docente de preescolar (2015-2016) y como terapeuta de aprendizaje de niños con rezago escolar (2012-2017). Obtuvo el grado de Magíster en Aprendizaje de la Lengua y las Matemáticas el 25 de noviembre de 2016, en la Universidad Autónoma de Querétaro (UAQ), México. Su tema de investigación se centra en conocer las ideas y recursos gráficos que niños de preescolar y primaria ponen en juego para marcar la diferencia entre palabras que son distintas por la presencia o ausencia de tilde.

Email: pausonil25@hotmail.com

Sofía Alejandra Vernon Carter es profesora de la Universidad Autónoma de Querétaro, Facultad de Psicología. En esta Universidad ha trabajado en los programas de Licenciatura en Psicología, en la Especialidad en Enseñanza y Aprendizajes Escolares y en la Maestría en Aprendizaje de la Lengua y las Matemáticas.

Email: sofiavernondocs@gmail.com

Para citar este artículo:

Soni Limón, P. y Vernon, S. A. (2018). La representación del acento en escritores principiantes muy jóvenes. Bellaterra Journal of Teaching \& Learning Language \& Literature, 11(2), 35-53. DOI: http://doi.org/10.5565/rev/jt13.770 